\title{
Plasmodium vivax CSP-Pvs25 variants from southern Mexico produce distinct patterns of infectivity for Anopheles albimanus versus An. pseudopunctipennis, in each case independent of geographical origin
}

Lilia González-Cerón ${ }^{1 *}$, Mario H. Rodríguez², José A. Nettel-Cruz, Juan E. Hernández-Ávilaª, lliana R. Malo-García, Frida Santillán-Valenzuela ${ }^{1}$ and Cuauhtémoc Villarreal-Treviño ${ }^{1 *}$

\begin{abstract}
Background: The susceptibility of Anopheles albimanus and An. pseudopunctipennis to local Plasmodium vivax has been associated in southern Mexico with two ookinete surface proteins (Pvs25/28) polymorphism. Perhaps parasite population selection (i.e. adaptation to local vectors) contributes to this phenomenon. It is also possible that certain molecular interactions exist between P. vivax and each mosquito species independently of geographical origin. This study aimed to explore the susceptibility of An. albimanus and An. pseudopunctipennis (collected from different geographical sites) to $P$. vivax cspVk/Pvs25-130 haplotypes from southern Mexico.

Results: Of the 120 P. vivax-infected blood samples used to simultaneously feed An. albimanus and An. pseudopunctipennis mosquitoes originating from various geographical sites, 80 produced at least one infected mosquito species. Three parasite haplotypes were identified in infected blood: Vk210/Pvs25-A (12.5\%), Vk210/Pvs25-B (20\%) and Vk247/Pvs25-B (67.5\%). Two parameters (the proportion of infected mosquitoes and number of oocysts/mosquito) showed a similar pattern for each mosquito species (independently of geographical origin). For An. albimanus mosquitoes (from the Pacific coast, Mexican gulf and Lacandon Forest lowlands), these two parameters were higher in specimens infected with $P$. vivax Vk210/Pvs25-A versus Vk210/Pvs25-B or Vk247/Pvs25-B $(P<0.001)$. For An. pseudopunctipennis mosquitoes (from the Pacific coast, northeast Mexico and east Guatemala foothills), the same two parameters were higher in specimens infected with Vk247/Pvs25-B or Vk210/Pvs25-B versus Vk210/Pvs25-A $(P<0.001)$. Higher infection rates were caused by Vk247/Pvs25-B than Vk210/Pvs25-B parasites in An. pseudopunctipennis $(P=0.011)$ and An. albimanus $(P=0.001)$. The greatest parasitaemia, gametocytaemia and microgamete formation was observed in Vk247/Pvs25-B infected blood, and each of these parameters correlated with each other and with the number of oocysts in An. pseudopunctipennis from the sympatric colony.

\footnotetext{
* Correspondence: Igonzal@insp.mx; cvilla@insp.mx

${ }^{1}$ Centro Regional de Investigación en Salud Pública, Instituto Nacional de

Salud Pública, Tapachula 30700, Chiapas, México

Full list of author information is available at the end of the article
}

(c) The Author(s). 2019 Open Access This article is distributed under the terms of the Creative Commons Attribution 4.0 International License (http://creativecommons.org/licenses/by/4.0/), which permits unrestricted use, distribution, and reproduction in any medium, provided you give appropriate credit to the original author(s) and the source, provide a link to the Creative Commons license, and indicate if changes were made. The Creative Commons Public Domain Dedication waiver (http://creativecommons.org/publicdomain/zero/1.0/) applies to the data made available in this article, unless otherwise stated. 


\begin{abstract}
(Continued from previous page)
Conclusions: Plasmodium vivax Vk247/Pvs25-B infections were the most prevalent, likely due to the higher parasitaemia produced in the susceptible vector (especially An. pseudopunctipennis). The analysis of mosquito-parasite interactions indicate that An. pseudopunctipennis and An. albimanus each have a unique pattern of transmitting genetic variants of $P$. vivax, and this is not dependent on geographical origin. The present findings highlight the importance of parasite genotyping to understand transmission dynamics and vectorial participation.
\end{abstract}

Keywords: Anopheles albimanus, Anopheles pseudopunctipennis, Susceptibility, Plasmodium vivax, Mexico, Guatemala, Circumsporozoite, Pvs25, Gametocytes,

\section{Background}

Anopheles albimanus Wiedemann, 1820 and An. pseudopunctipennis Theobald, 1901 are the main malaria vectors in Mexico, Central America and northern South America [1]. The former occurs on the coastal plains and in other low-altitude regions from southern USA to northern Peru and the Caribbean Islands [2]. Although no subgroups were revealed by polytene chromosome analysis and cross hybridization [3], differences in allozyme patterns [3] and in ribosomal DNA structure [4] have been documented. An. pseudopunctipennis is found at altitudes over $200 \mathrm{~m}$ above sea level from southern USA to northern Argentina [5]. Three population clusters have been identified by isozyme analysis, one in southern USA-Guatemala region, another distributed from South America to Central America, and the third in the Antilles [5].

In the last decade, Plasmodium vivax has produced more than $99 \%$ of malaria cases in Mexico [6, 7]. The known difference between the two $P$. vivax variants lies in the repeat units (Vk) of the circumsporozoite protein (CSP): Vk210 (GRA[A/D]GQPA) [8] and Vk247 (ANGAGNQPG) [9]. Experimental infections using local mosquitoes and infected blood from patients in the Soconusco region (in southern Mexico) showed An. albimanus to be more susceptible to Vk210 parasites and An. pseudopunctipennis to Vk247 [10]. Variant Vk210 is more prevalent in patients from the coastal plains (where An. albimanus predominates), and variant Vk247 in patients from the foothills (where An. pseudopunctipennis predominates).

Further studies ascertained the identity and location of the Pvs25 and Pvs28 ookinete surface protein polymorphs. Parasites from the coastal plains were Vk210 with Pvs25 87 Gln/130 Ile and Pvs28 87 Asn/110 Tyr (Pvs25-A/Pvs28-A), identical to those of the Sal I reference strain [11] and infective only for An. albimanus [12]. Parasites from the foothills were either Vk210 or Vk247, associated with Pvs25 and displaying substitutions at 130 Thr and Pvs28 87 Asp/110 Asn (Pvs25-B and Pvs28-B), and the Pvs25 substitution 87 Gln-Lys subdivided parasites into B1 or B2. They were more infective for An. pseudopunctipennis [12]. An association between cspVk variants of parasites and their local vectors was supported by microsatellite analysis, which provided evidence that $P$. vivax populations are structured according to their sympatric vector distribution. Two related subpopulations were detected in the foothills [13], presumably expressing Pvs25/28 type B [12]. These observations indicate the adaptation and possible selection of parasite variants in each geographical area in accordance with the predominant vector.

The distribution of An. albimanus and An. pseudopunctipennis along the extensive malarious areas of Mexico follows the pattern reported in the southern part of the State of Chiapas. Although the possible existence of subpopulations of the same mosquito species has not been examined in detail, geographical isolation could favor the selection of some distinguishing characteristics $[3,4]$. For instance, there is a different degree of susceptibility to sympatric and allopatric $P$. vivax isolates for An. albimanus from El Salvador and Panama [14]. Thus, the association between cspVk/Pvs25 haplotypes and anopheline vectors documented in Chiapas may not occur outside this area.

The aim of the present study was to investigate the susceptibility of An. albimanus and An. pseudopunctipennis, collected from various geographical sites, to $P$. vivax cspVk/Pvs25 haplotypes from southern Mexico. The results, similar to those previously found in southern Mexico, suggest that distinctive molecular interactions take place between a given parasite haplotype and each mosquito species.

\section{Methods}

\section{Mosquito collection and colonization}

Batches of about 750-1300 larvae and/or adult An. pseudopunctipennis and/or An. albimanus mosquitoes were gathered in Mexico from Lacandon Forest in Chiapas, the Pacific coast regions of the states of Chiapas and Oaxaca, and the Gulf coast areas of the states of Veracruz and Nuevo Leon. Specimens of An. pseudopunctipennis were also taken in Zacapa, Guatemala (Fig. 1, Additional file 1: Table S1). In Mexico, the study regions have been historically affected by malaria transmission, but at present malaria is focused on the Pacific coast and along the border with Guatemala $[6,7]$. In 


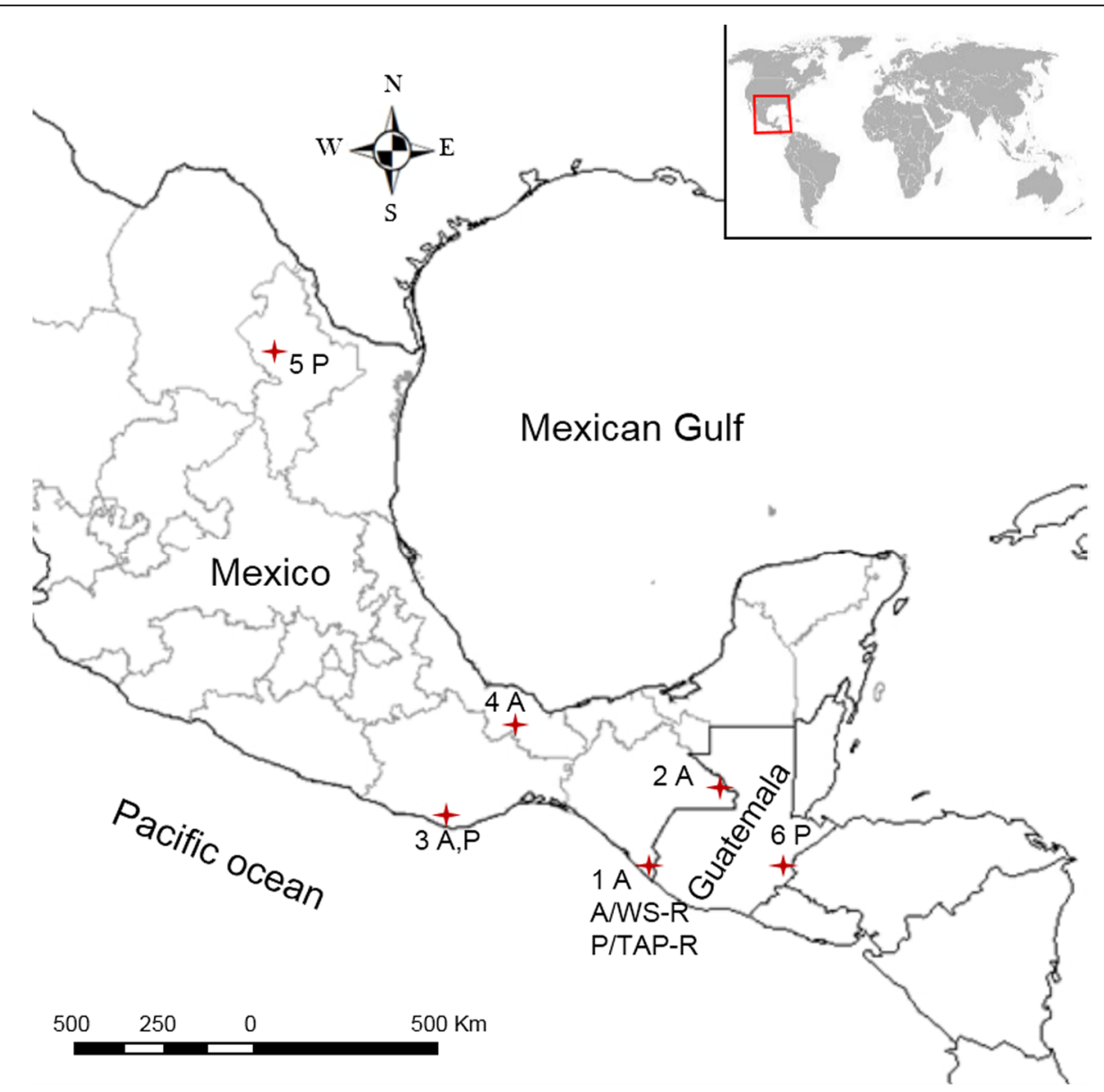

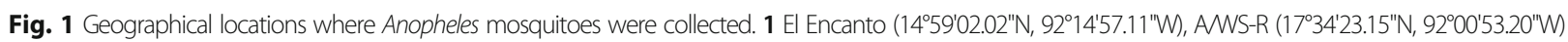

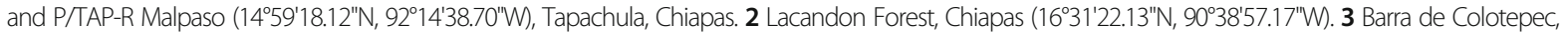

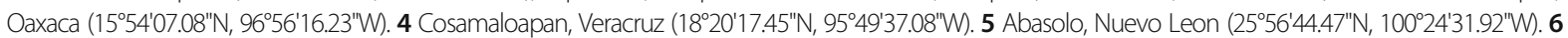
Zacapa, Guatemala (1458'42.93"N, 89³1'39.50"W). Abbreviations: A, An. albimanus; P, An. pseudopunctipennis; ANS-R, reference strain of An. albimanus; P/TAP-R, reference strain of An. pseudopunctipennis

Guatemala, malaria transmission occurs in most of the country including Zacapa, but the highest number of cases are reported in Escuintla and Alta Verapaz Departments [6].

Adult female mosquitoes were captured while resting in bovine livestock corrals, and larvae were collected from breeding sites with standardized methodologies [15]. Mosquitoes were transported to the Regional Centre for Research in Public Health (CRISP)/INSP for the development of the colonies under insectary conditions. Each batch of larvae and adult mosquitoes was labeled with the date of capture and georeferenced to indicate the town, municipality and state. The species of adult mosquitoes was determined by employing morphological-based keys [16]. Larvae were maintained in trays with water until adult mosquitoes emerged. Adults obtained from larvae were used to establish colonies classified by geographical origin. Mosquito copulation was induced as previously described [17]. Each colony was kept in a different room, and trays were covered with mosquito netting to avoid crossing or contamination between mosquitos from distinct regions. Adult mosquitoes were sustained with cotton impregnated with $5 \%$ sugar in water and fed on rabbit blood in artificial membrane feeders. Rabbits were raised and handled according to the Mexican guidelines for laboratory animals [18]. Adult mosquito cages were individually handled to avoid cross contamination between colonies.

The colonies were considered sufficiently stable when natural sexual encounters without any external stimulus resulted in enough females to sustain reproduction (at about the 4th to 16th generations) (Additional file 1: Table S1). Colonies of the white-striped phenotype of An. albimanus (A/WS-R) and the Tapachula strain of An. pseudopunctipennis (P/TAP-R) served as references [10]. The A/WS-R colony was developed in the late 1990 s from mosquitoes gathered in the Soconusco region, the southern-most tip of Chiapas, Mexico. This strain is highly susceptible to $P$. vivax Vk210/Pvs25-A 
(refers to Pvs25-130Ile) $[12,19]$ and almost refractory to either P. vivax Vk247 or Vk210 and Pvs25-B (refers to Pvs25-130Thr) [12]. The P/TAP-R colony, raised in 1995 from larvae collected in Malpaso (Tapachula, Chiapas) [17], was very susceptible to $P$. vivax Vk247 and Vk210 expressing Pvs25-B but almost resistant to Vk210/ Pvs25-A [12]. Groups of 50-200 females (2-5 days post-emergence) of colonized An. albimanus and $A n$. pseudopunctipennis from the diverse geographical sites were used in susceptibility assays (Additional file 1: Table S1).

\section{Plasmodium vivax-infected blood samples}

With prior informed consent, $P$. vivax-infected 5 -ml blood samples were obtained from 120 symptomatic patients living on the coast and in the foothills of the Soconusco region. Participating and non-participating patients were treated immediately with chloroquine and primaquine, according to the Mexican guidelines [20].

Parasitaemia, gametocytaemia and microgamete formation Microgamete formation was quantified by in vitro exflagellation assays (in duplicate) with fresh infected blood. Briefly, immediately after each blood sample was drawn, $10 \mu \mathrm{l}$ were mixed with $400 \mu \mathrm{l}$ of cold RPMI medium plus $0.05 \mathrm{mg} / \mathrm{ml}$ hypoxanthine at $\mathrm{pH} 8.3$ (Sigma-Aldrich, St. Louis, MO, USA). Ten microliters of each sample was mounted under coverslips and incubated for $15 \mathrm{~min}$ at room temperature (about $24{ }^{\circ} \mathrm{C}$ ) [21]. The total number of rosettes (exflagellations) per preparation were counted under microscope at $400 \times$ magnification, and the number of exflagellations per $\mu \mathrm{l}$ was calculated. The complete procedure was accomplished in $30 \mathrm{~min}$. Thick blood smears were prepared and Giemsa stained, as were thin blood smears fixed with methanol. For each sample, the total parasitaemia and gametocytaemia were evaluated by two skilled microscopists who counted fields with up to 500 white blood cells at $1000 \times$ magnification. For the calculation of the number of parasites per $\mu \mathrm{l}$ of infected blood, the average number of white cells/ $\mu$ l of blood was considered to be 7000 [22].

Preparation of Plasmodium vivax-infected blood for feeding Each infected blood sample was centrifuged at $3000 \mathrm{rpm}$ for $5 \mathrm{~min}$ and the plasma removed. Blood cells were washed with phosphate buffer at pH 7.2 and $37{ }^{\circ} \mathrm{C}$ to delay gamete formation [23] and then centrifuged as above. After discarding the washing solution, blood cells were re-suspended in $40 \%$ hematocrit with a non-immune serum of the same blood type, as previously described [10].

\section{Mosquito feeding, maintenance and infection}

The feeding experiments consisted of batches of female mosquitoes of each species from distinct sites and the reference colonies (A/WS-R and P/TAP-R). Female mosquitoes of generations 6-76 (Additional file 1: Table S1) were deprived of sugar-impregnated cotton for $2-6 \mathrm{~h}$ before being simultaneously exposed to $P$. vivax infected blood (provided through artificial feeders) for 45-60 min. After the blood-feeding, unfed mosquitoes were removed to maintain only the engorged insects under insectary conditions $\left(24-27{ }^{\circ} \mathrm{C}, 70-85 \%\right.$ humidity). At 7-8 days post-feeding, groups of up to 25 mosquitoes from each experimental colony were examined and their midguts were dissected and treated with mercurochrome to stain the oocysts [10]. The proportion of mosquitoes with at least one oocyst and the number of oocysts per mosquito were recorded.

\section{Plasmodium vivax genotyping}

Parasites from blood samples that produced infection in at least one mosquito colony were analyzed for the CSP repeat type and the Pvs25-residue 130 [12]. Briefly, DNA obtained from the infected blood samples was scrutinized for the cspVk by PCR amplification and hybridization, using specific probes as reported [10]. For Pvs25, a gene fragment that had the residue of interest (codon 130) was amplified with primers Pvs25-F23 (nt129-149) 5'-GTG TAT GTG TAA CGA AGG GCT-3' and Pvs25-R214 (nt 453-472) 5'-CAG TTT CTC CCG TTT TGG TA-3'. The amplification mixture contained $100 \mathrm{ng}$ of total genomic DNA, $0.25 \mathrm{mmol}$ of each primer, $1 \times \mathrm{GoTaq}^{\circledR}$ flexi buffer ( $\mathrm{pH} 8.5$ ), $1.5 \mathrm{mmol}$ $\mathrm{MgCl} 2,0.6 \mathrm{mmol} \mathrm{dNTPs}$ and $1.5 \mathrm{U}$ of $\mathrm{GoTaq}^{\bullet}$ Flexi DNA polymerase (Promega, Madison, WI, USA). PCR conditions were: $95{ }^{\circ} \mathrm{C}$ for $3 \mathrm{~min}$ followed by 35 cycles of $95{ }^{\circ} \mathrm{C}$ for $45 \mathrm{~s}, 60{ }^{\circ} \mathrm{C}$ for $45 \mathrm{~s}, 68{ }^{\circ} \mathrm{C}$ for $1 \mathrm{~min}$, and 72 ${ }^{\circ} \mathrm{C}$ for 5 min using a T-100 Thermal Cycler (BioRad, Hercules, CA, USA). Two samples that did not amplify were treated with primers Pvs25-F22 (nt268-289) 5'-ATG TAC AAA TGT GGT TGC ATT G-3' and Pvs25-R214; with these primers the amplification was more efficient. The aforementioned amplification mixture and conditions were employed.

Subsequently, PCR products of the expected molecular size were purified with the Wizard DNA Clean-Up System (Promega) according to the manufacturer's instructions, and sequenced at the High Throughput Genomics Unit (Department of Genome Sciences, University of Washington, Seattle, WA, USA) or at Macrogen Inc. (Seoul, Republic of Korea). The sequences were aligned by using the homologous sequence of the Sal I genome (XM_001608410.1) and the variation at codon 130 was recorded.

\section{Statistical analysis}

The quantitative variables (i.e. parasitaemia, gametocytaemia and the number of exflagellations) were compared 
with the Kruskal-Wallis test by ranks [24]. Differences in the proportion of infected mosquitoes were analyzed with logistic regression. The intensity of infection (number of oocyst per infected mosquito) was compared between blood samples with a negative binomial model [25]. These models are suitable for data not showing normal distribution. We assumed that the proportion of infected mosquitoes had a binomial distribution and the oocyst count per infected mosquito a negative binomial distribution. In both models, the species/origin was coded as an 8-level factor. The csp repeat type (Vk210 or Vk247) and Pvs25 residue 130 Ile (Pvs25-A)/Thr (Pvs25-B) were coded as a 3-level factor: Vk247/Pvs25-B, Vk210/Pvs25-A and Vk210/Pvs25-B as reported earlier [12]. The fitted model included all main effects and was examined based on two-way interactions. The statistical significance of the differences was estimated by the linear combination of the regression coefficients, for both the proportion of infected mosquitoes and intensity of infection. The variance of the coefficients was evaluated with the Jackknife variance estimator [26, 27]. A separate logit model was fitted to test the difference in simultaneous infections of An. pseudopunctipennis with haplotypes Vk210/Pvs25-B and Vk247/ Pvs25-B. The mosquito colonies originating from Guatemala and Nuevo Leon (Mexico) were compared to the reference. The association between parasite blood parameters and the outcome of the susceptible vector was evaluated with Spearman's rank correlation coefficient [28]. All significance tests were carried out at the 95\% confidence level with Stata v.9.2 Statistical Package (Stata Co. 2006).

\section{Results}

\section{Plasmodium vivax-infected blood: parasite counts and} haplotypes

In $111 P$. vivax feeding assays sufficient mosquitoes were fed and survived up to day 7 for midgut inspection, and in 80 of them oocyst infection in at least one mosquito colony was detected. Ten of these resulted from infected blood with haplotype Vk210/Pvs25-A, taken from patients living on coastal plains. Among the other 70 infections, triggered by the blood of patients living in foothills, 16 were of haplotype Vk210/Pvs25-B and 54 of haplotype Vk247/Pvs25-B. Blood infected with Vk247/ Pvs25-B had higher parasitaemia than that infected with Vk210/Pvs25-B (Kruskal-Wallis test, $\chi^{2}=8.42, d f=1$, $P=0.003)$ and had higher gametocytemia than blood infected with Vk210/Pvs25-B (Kruskal-Wallis test, $\chi^{2}=$ 4.31, $d f=1, P=0.037$ ) or Vk210/Pvs25-A (Kruskal-Wallis test, $\chi^{2}=5.23, d f=1, P=0.022$ ) (Table 1 ). No difference in the rate of exflagellation was found between parasite haplotypes (Kruskal-Wallis test, $\chi^{2}=4.88, d f=2$, $P=0.086)$.
The majority of blood samples were infected with haplotype Vk247/Pvs25-B (67.5\%). The 49 experiments that had data from all blood parameters showed that parasitaemia was highly correlated with gametocytaemia (Spearman's correlation, $\rho=0.75, d f=48, P<0.001$ ) (Fig. 2a). The correlation of parasitaemia and gametocytaemia with the rate of exflagellation was also significant (Spearman's correlation: $\rho=0.52, d f=48, P<0.001$ and $\rho=0.45, d f=48, P=0.001$, respectively) (Fig. $2 \mathrm{~b}, \mathrm{c}$ ). For Vk210/Pvs25-B (20\% of the samples), a correlation was only observed between parasitaemia and gametocytaemia (Spearman's correlation, $\rho=0.75, d f=15, P=0.001$ ). For the few Vk210/Pvs25-A samples, no correlation existed between any of the parasite blood parameters.

\section{Proportion of infection by the $P$. vivax haplotypes in each of the two mosquito species}

There was a highly consistent susceptibility pattern for each mosquito species (An. albimanus and An. pseudopunctipennis) to the different $P$. vivax haplotypes. This pattern was independent of the geographical origin of the mosquito colonies.

The proportion of infected An. albimanus mosquitoes (from all sites) was 17.35 times higher (95\% CI: 12.74-23.64) when fed with blood containing $P$. vivax Vk210/Pvs25-A versus Vk210/Pvs25-B or Vk247/Pvs25-B (logistic model Wald Test, $t=18.1, d f=2247, P=0.001)$. The proportion of infected mosquitoes from the coast of Chiapas and Veracruz fed with Vk210/Pvs25-B was lower than that of the reference colony (A/WS-R). Similarly, the proportion of infected mosquitoes from Oaxaca and the Lacandon Forest (Chiapas) fed with Vk247/Pvs25-B was lower than that of the reference colony (Table 2).

Likewise, the proportion of infected An. pseudopunctipennis mosquitoes (from all sites) was 54.03 times higher (95\% CI: 29.94-97.48) when fed with P. vivax Vk247/ Pvs25-B or Vk210/Pvs25-B versus Vk210/Pvs25-A (logistic model Wald test, $t=13.26, d f=1655, P<0.001$ ). Feeding with $P$. vivax Vk247/Pvs25-B or Vk210/Pvs25-B caused a 19.27 times higher proportion of infected $A n$. pseudopunctipennis than infected An. albimanus mosquitoes (95\% CI: 16.10-23.07; logistic model Wald Test, $t=32.25, d f=1379, P<0.001)$. For each parasite haplotype, the proportion of infected mosquitoes in the $A n$. pseudopunctipennis colonies (from almost all sites) was similar to that observed in the reference. The only exception was the colony from Guatemala, which had a lower proportion of infected mosquitoes than the reference (logistic model Wald test, $t=-2.43, d f=976$, $P=0.015)$ (Table 2).

When only simultaneous infections with the same parasite haplotype were compared to the reference mosquito colony, the proportion of infected individuals was lower in the allopatric mosquito colonies originating from Nuevo 
Table 1 Plasmodium vivax infected blood: parasitaemia, gametocytaemia and exflagellation rates for each parasite genotype

\begin{tabular}{|c|c|c|c|c|c|}
\hline $\begin{array}{l}\text { Blood infection with } P \text {. vivax genotypes: cspVK/ } \\
\text { Pvs } 25-130\end{array}$ & $n$ & $\begin{array}{l}\text { Median (IQR) of parasites/ } \\
\mu \text { of blood }\end{array}$ & $\begin{array}{l}\text { Median (IQR) of gametocytes/ } \\
\mu \text { l of blood }\end{array}$ & $n$ & $\begin{array}{l}\text { Median (IQR) of exflagellations/ } \\
\mu \text { of blood }\end{array}$ \\
\hline Vk210/Pvs25-A & 9 & $5040(4400-6960)$ & $225(113-288)$ & 10 & $36(12-116)$ \\
\hline Vk210/Pvs25-B & 16 & $4360(2800-6000)$ & $218(116-628)$ & 16 & $18(4-50)$ \\
\hline Vk247/Pvs25-B ${ }^{b}$ & 54 & $8000(4640-14,640)$ & $617(259-1120)$ & 50 & $42(20-152)$ \\
\hline
\end{tabular}

Notes: Parasitaemia was higher with genotype Vk247/Pvs25-B versus Vk210/Pvs25-B $(P=0.003)$. Gametocytaemia was higher with genotype Vk247/Pvs25-B versus Vk210/Pvs25-A $(P=0.022)$ or Vk210/Pvs25-B $(P=0.037)$. There were no differences in the number of exflagellations between genotypes $(P=0.086)$. Kruskal-Wallis test by ranks $(95 \% \mathrm{Cl})$

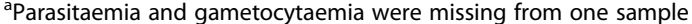

${ }^{\mathrm{b}}$ The exflagellation values were missing from four samples

Abbreviation: $n$, number of parasite isolates

Leon and Guatemala (Table 3). For P. vivax Vk210/ Pvs25-B, the proportion of infected mosquitoes in the Nuevo Leon colony was 0.53 and in the Guatemala colony 0.50 , compared to the 0.76 found in P/TAP-R colony (logistic model Wald Test: $t=-2.23, d f=153, P=0.027$ and $t=-2.57, d f=153, P=0.011$, respectively). Nuevo Leon (Mexico) is about $2000 \mathrm{~km}$ from Tapachula, the home of the P/TAP-R colony, and the Guatemala collection site is about $440 \mathrm{~km}$ away. There was no difference in the proportion of infection between the P/TAP-R, Guatemala and Oaxaca colonies. For Vk247/Pvs25-B parasites, the only significant difference from P/TAP-R proportion (0.79) was represented by the colony originating from Nuevo Leon (0.65) (logistic model Wald test, $t=-2.14, d f=321, P=0.030$ ) (Table 3).

Overall, a 1.4 times higher proportion of both An. pseudopunctipennis and An. albimanus were infected when fed
Vk247/Pvs25-B versus Vk210/Pvs25-B parasites (logistic model Wald test, $t=4.51, d f=3379, P<0.001$ ) (Table 4). Considering only An. pseudopunctipennis mosquitoes, the difference in the proportion of infected mosquitos between these two haplotypes ranged from 0.15 to 0.24 (Table 2).

\section{Oocyst density generated by $P$. vivax cspVk/Pvs25-130} haplotypes in An. albimanus and An. pseudopunctipennis Comparing only infected An. albimanus mosquitoes, a significantly higher oocyst count was found in those infected with the Vk210/Pvs25-A versus Vk247/Pvs25-B (negative binomial model Wald test, $t=2.99, d f=396, P=0.003$ ) (Table 2). Although the number of oocysts was slightly greater in mosquitoes infected with the Vk210/Pvs25-B versus Vk247/Pvs25-B or lower with the Vk210/Pvs25-A versus Vk210/Pvs25-B haplotypes, the difference was not
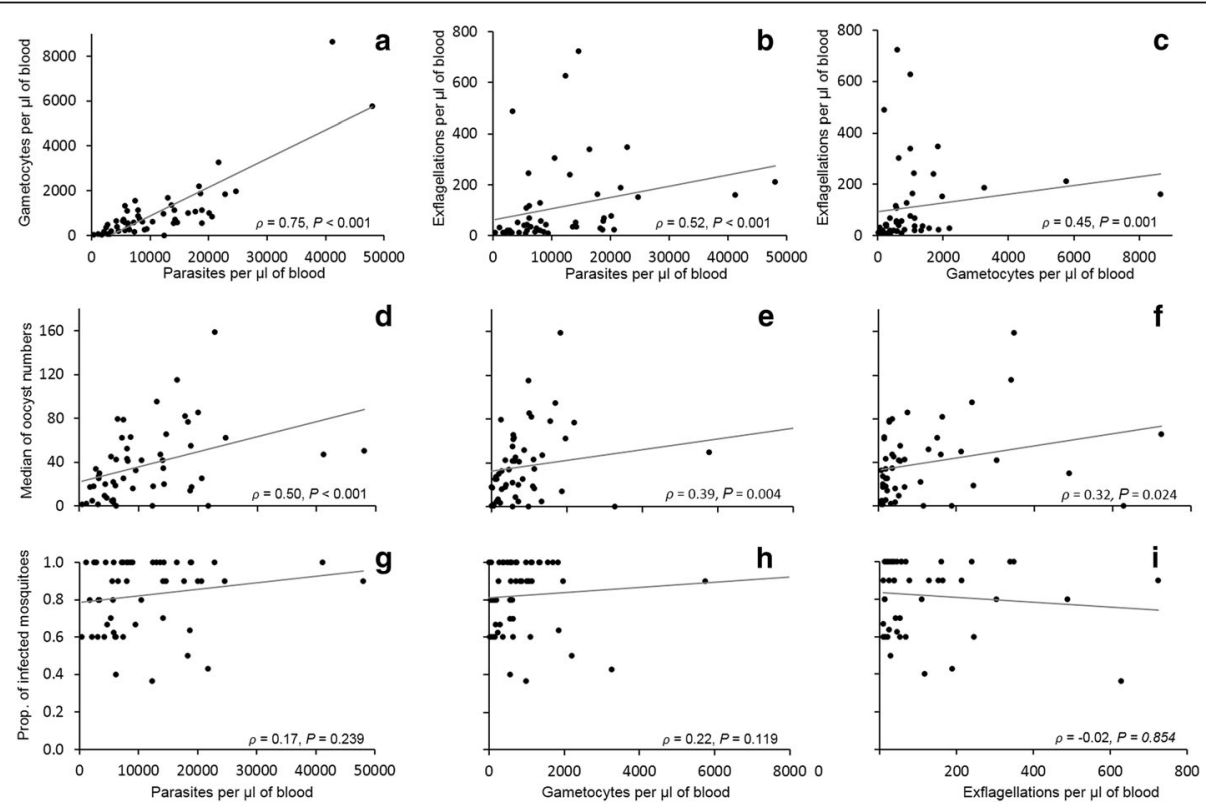

Fig. 2 Relationship between P. vivax Vk247/Pvs25-B blood parameters and Anopheles pseudopunctipennis infection outcomes. Scatter graphs are shown with a regression line. a-c The correlation is portrayed between $P$. vivax blood parameters: parasitaemia, gametocytaemia and the number of exflagellations. $\mathbf{d}$-f The relation between the data of blood parameters and the median number of oocysts developed in susceptible mosquitoes is depicted. $\mathbf{g}$-i The lack of association between blood parameters and the proportion of infected mosquitoes can be appreciated. Spearman's correlation outputs are indicated in each graph (at 95\% Cl) 
Table 2 The proportion of An. albimanus and An. pseudopunctipennis mosquitoes (from various geographical sites) infected with oocysts and the corresponding oocyst density produced by different P. vivax genotypes (cspVk /Pvs25-130) from southern Mexico

\begin{tabular}{|c|c|c|c|c|c|c|c|c|c|}
\hline \multirow{2}{*}{$\begin{array}{l}\text { Geographical site and } \\
\text { mosquito colony }\end{array}$} & \multicolumn{3}{|c|}{ Vk210/Pvs25-A } & \multicolumn{3}{|c|}{ Vk210/Pvs25-B } & \multicolumn{3}{|c|}{ Vk247/Pvs25-B } \\
\hline & $n$ & Prop. infected & $\begin{array}{l}\text { Median number } \\
\text { of oocysts (IQR) }\end{array}$ & $n$ & Prop. infected & $\begin{array}{l}\text { Median number } \\
\text { of oocysts (IQR) }\end{array}$ & $n$ & Prop. infected & $\begin{array}{l}\text { Median number } \\
\text { of oocysts (IQR) }\end{array}$ \\
\hline \multicolumn{10}{|l|}{ An. albimanus } \\
\hline 1. AMS-R & 210 & 0.72 & $14(6.0-28.0)$ & 280 & 0.10 & $7(4.0-17.0)$ & 1133 & 0.17 & $4(1.0-15.0)$ \\
\hline 1. Coast, Chiapas & - & - & - & 65 & $0.0 \stackrel{0.001}{0}$ & $15.0(15.0-15.0)$ & 70 & $0.11^{0.285}$ & $4(2.5-5.0)$ \\
\hline 2. LF, Chiapas & 10 & $0.40^{0.067}$ & $5(2.5-10.0)$ & 45 & $0.07^{0.519}$ & $2.0(2.0-17.0)$ & 205 & $0.03 \leq 0.001$ & $9(3.0-10.0)$ \\
\hline 3. Oaxaca & 10 & $0.80^{0.690}$ & $18.5(7.0-25.0)$ & 56 & $0.04^{0.260}$ & $7.0(6.0-8.0)$ & 119 & $0.01 \stackrel{0.001}{=}$ & $66(66.0-66.0)$ \\
\hline 4. Veracruz & 10 & $0.40^{0.067}$ & $11(4.0-23.5)$ & 20 & 0.0 & - & 35 & $0.06^{0.233}$ & $3.5(1.0-6.0)$ \\
\hline \multicolumn{10}{|l|}{ An. pseudopunctipennis } \\
\hline 1. P/TAP-R & 113 & 0.03 & $2(1.0-3.0)$ & 147 & 0.60 & $17(7.0-39.0)$ & 415 & 0.80 & $41(17.0-72.0)$ \\
\hline 3. Oaxaca & 28 & $0.07^{0.398}$ & $12.5(9.0-16.0)$ & 87 & $0.55^{0.552}$ & $16.5(7.0-39.5)$ & 80 & $0.79^{0.765}$ & $33(21.0-57.0)$ \\
\hline 5. Nuevo Leon & 75 & $0.03^{0.997}$ & $1(1.0-1.0)$ & 59 & $0.53^{0.391}$ & $19(8.0-41.0)$ & 275 & $0.81^{0.876}$ & $39(18.0-63.0)$ \\
\hline 6. Guatemala & 68 & $0.09^{0.135}$ & $1.5(1.0-3.0)$ & 100 & $0.56^{0.622}$ & $20.5(8.5-36.5)$ & 207 & $0.71 \stackrel{0.015}{0}$ & $27(10.5-53.0)^{\mathrm{a}}$ \\
\hline
\end{tabular}

Notes: Only infected mosquitoes were considered for the calculation of the median number of oocysts and its IQR. $P$-values from the comparison of the proportion of infected mosquitoes of a colony against the reference strain are indicated as superscripts (underlined are those having significance)

${ }^{a}$ Compared to the reference mosquitoes, the number of oocysts was significantly lower in the Guatemala colony $(P=0.002)$ when fed with parasite genotype Vk247/Pvs25-B. Statistical significance was calculated by using the Jackknife coefficient variance estimator at $95 \%$ confidence interval (according to models described in method section)

Abbreviations: $n$ total number of mosquitoes examined, Prop. proportion, A/WS-R reference strain of An. albimanus, P/TAP- $R$ reference strain of An. pseudopunctipennis, LF Lacandon Forest, IQR interquartile range

significant. The few A/WS-R An. albimanus infected with Vk210/Pvs25-B or Vk247/Pvs25-B parasites had a small number of oocysts in their midguts. Because the proportion of An. albimanus mosquitoes from most other colonies infected with these two parasite haplotypes was also very small, no statistical analysis could be performed.

In assays with $A n$. pseudopunctipennis, the intensity of oocyst infection was 8.54 (95\% CI: 3.65-19.99) times higher with Vk210/Pvs25-B than with Vk210/Pvs25-A parasites (negative binomial model Wald test, $t=4.95$, $d f=1002, P<0.001)$ and 13.27 (95\% CI: 5.73-30.76) times higher when infected with Vk247/Pvs25-B (negative binomial model Wald test, $t=6.04, d f=1002, P<0.001$ ) (Tables 2, 3). As an exception, two mosquitoes from the Oaxaca colony were infected with the Vk210/Pvs25-A

Table 3 Comparison of the mosquitoes infected by different $P$. vivax genotype (cspVk/Pvs25-130) only from simultaneous feeding of An. pseudopunctipennis from different geographical sites

\begin{tabular}{|c|c|c|c|c|c|c|}
\hline \multirow{2}{*}{$\begin{array}{l}\text { Geographical site and } \\
\text { mosquito colony }\end{array}$} & \multicolumn{3}{|c|}{ Vk210/Pvs25-B ${ }^{a}$} & \multicolumn{3}{|c|}{ Vk247/Pvs25-B } \\
\hline & $n$ & $\begin{array}{l}\text { Prop. } \\
\text { infected }\end{array}$ & P & $n$ & $\begin{array}{l}\text { Prop. } \\
\text { infected }\end{array}$ & P \\
\hline 1. P/TAP-R & 49 & 0.76 & & 107 & 0.79 & \\
\hline 5. Nuevo Leon & 49 & 0.53 & 0.027 & 109 & 0.65 & 0.030 \\
\hline 6. Guatemala & 56 & 0.50 & 0.011 & 106 & 0.72 & 0.260 \\
\hline
\end{tabular}

Notes: Statistical significance was calculated by using the Jackknife coefficient variance estimator at $95 \%$ confidence interval

${ }^{a}$ Six simultaneous experiments

${ }^{\mathrm{b}}$ Seven simultaneous experiments

Abbreviations: $n$, total number of mosquitoes examined; Prop., proportion; $P /$

$T A P-R$, reference strain of An. pseudopunctipennis haplotype and had 9 and 16 oocysts in their midgut, respectively. The infected blood used in this assay had the second highest parasitaemia and the highest gametocytaemia and number of rosettes among the Vk210/Pvs25-A parasite lots. The mean number of oocysts was significantly lower in mosquitoes from Guatemala, Nuevo Leon and P/TAP-R colonies with $P$. vivax Vk210/Pvs25-A versus Vk247/Pvs25-B or Vk210/Pvs25-B (Table 2). Compared to the reference colony, Vk247/Pvs25-B parasites generated significantly lower oocyst densities in An. pseudopunctipennis from Guatemala (negative binomial model Wald test, $t=-2.99, d f=765, P=0.003$ ) (Table 2).

\section{Correlation between $P$. vivax blood parameters and sympatric mosquito infections}

The Vk247/Pvs25-B blood infection parameters correlated with the median number of oocysts in An. pseudopunctipennis (P/TAP-R). The number of oocysts showed higher correlation with parasitaemia (Spearman's correlation, $\rho=0.50$, $d f=48, P<0.001$ ) than with gametocytaemia (Spearman's correlation, $\rho=0.39, d f=48, P=0.004$ ) or exflagellation rates (Spearman's correlation, $\rho=0.32, d f=48, P=0.024$ ) (Fig. 2d, f). In contrast, none of the blood parasite parameters correlated with the proportion of infected mosquitoes (Fig. 2g, i).

Compared to $P$. vivax Vk247/Pvs25-B, fewer assays were conducted with Vk210/Pvs25-B and Vk210/Pvs25-A. With the latter haplotypes, there was no correlation between blood parameters and the median number of oocysts or the proportion of infected mosquitoes, either for 
Table 4 Differential susceptibility of An. albimanus and An. pseudopunctipennis mosquitoes to two related $P$. vivax genotypes

\begin{tabular}{llll}
\hline$P$. vivax (cspVk/Pvs25-130) & $n$ & Prop. infected & Median oocyst number (IQR) \\
\hline & An. albimanus & & $7(3-17)$ \\
Vk210/Pvs25-B & 466 & 0.075 & $4(1-14) ; P=0.100$ \\
Vk247/Pvs25-B & 1542 & $0.132 ; P=0.001$ & $18(7-39)$ \\
& An. pseudopunctipennis & & $36(16-62) ; P<0.001$ \\
Vk210/Pvs25-B & 393 & 0.56 & $0.784 ; P=0.011$ \\
Vk247/Pvs25-B & 979 & .
\end{tabular}

Notes: The median number of oocysts was calculated only considering the infected mosquitoes. Statistical significance was estimated by using the Jackknife coefficient variance estimator at $95 \%$ confidence interval

Abbreviations: $n$, total number of mosquitoes examined; Prop. proportion, $I Q R$ interquartile range

An. pseudopunctipennis or An. albimanus. A low exflagellation rate $(n=21$, median $=4, \mathrm{IQR}=0-12)$ was the only blood parasite parameter associated with the absence of oocyst infection in both vector species versus the development of oocysts in at least one species $(n=80$, median $=36$, IQR $=14-116)$ (Kruskal-Wallis test, $\chi^{2}=26.42, d f=1$, $P<0.001)$.

\section{Discussion}

The present results confirm our previous findings on different infectivity of $P$. vivax ookinete cspVk/Pvs25 variants for sympatric An. albimanus and An. pseudopunctipennis in southern Mexico [12]. Anopheles albimanus and An. pseudopunctipennis mosquitoes from several geographical origins in Mexico (as well as An. pseudopunctipennis from nearby Zacapa, Guatemala) were herein exposed to blood with distinct $P$. vivax haplotypes (Vk210/Pv25-A; Vk210/Pvs25-B and Vk247/ Pvs25-B). Each of the two mosquito species showed a distinct pattern of susceptibility to the haplotypes, which was consistent for the various geographical locations. Although only parasites from southern Mexico were used to infect mosquitoes, these observations suggest the existence of similar molecular mechanisms for the parasite-mosquito interaction when considering a particular parasite variant and mosquito species from various sites. This evidence points to the probability that parasite variant selection was not the only factor determining the increased infectivity of the parasites to sympatric mosquitoes.

No distinctive populations of An. albimanus have been identified, but differences in allozyme patterns [3] and ribosomal DNA structure [4] are indicative of possible early divergence between geographically isolated populations; whereas, three clusters of An. pseudopunctipennis were established by isozyme analysis distributed in Mexico, Central and South America [5] and two lineages of An. pseudopunctipennis were recently detected in Colombia [29]. Overall, these reports evidence the existence of diverse populations of the two vector species included presently.
Data on the susceptibility of these two anopheline species from different malarious areas is inconclusive. Anopheles pseudopunctipennis has been recognized as one of the principal malaria vectors throughout its geographical distribution from Mexico to northern South America [1, 30-33]. However, research on the susceptibility of this anopheline species suggest that it serves as a poor vector for the Central American Sal I and II strains [34]. The involvement of An. albimanus in $P$. vivax transmission was first examined decades ago in Central America [14, 35, 36]. Further studies documented that this vector is highly adapted to transmit parasite strains from this region, but showed low susceptibility to $P$. vivax from other geographical sites $[37,38]$. Using cspVk variants as markers, field-captured $A n$. albimanus displayed infection with $P$. vivax Vk210 and Vk247 at similar rates in Belize [39], but in Colombia, a higher number of sporozoites was detected in the salivary glands of An. albimanus when infected with $P$. vivax Vk247 than Vk210 parasites.

Still using cspVk variants as markers, we previously reported differences in southern Mexico between the susceptibility of An. albimanus and An. pseudopunctipennis to $P$. vivax Vk210 from the coastal regions and to $\mathrm{Vk} 247$ from the foothills [10]. We later evidenced that mosquito susceptibility was determined by the capacity of the parasites to invade the midgut epithelium and that ookinetes were unable to invade the midgut epithelium or were destroyed during invasion [40, 41]. Since the production of CSP protein begins in sporoblasts, this molecule plays no role during parasite invasion and the establishment of midgut infections. In further studies, Pvs25 and Pvs28 ookinete surface proteins, conforming haplotypes with cspVk variants, proved to be associated with parasite midgut invasion [12]. The predominant parasite haplotype in coastal areas was Vk210/Pvs25-A, while those in the foothills were Vk210/Pvs25-B and Vk247/Pvs25-B. Parasites with Pvs25-A were more infective for An. albimanus and those with Pvs25-B for An. pseudopunctipennis [12]. This pattern of susceptibility for these two mosquito species was presently 
exhibited independently of their geographical origin. However, because of the small numbers of mosquitoes tested, our data are not strictly conclusive but suggest that An. albimanus is highly susceptible to $P$ vivax Vk210/Pvs25-A in the study sites.

Interestingly, higher infection rates were produced by the parasite haplotypes Vk210/Pvs25-B and Vk247/ Pvs25-B in sympatric An. pseudopunctipennis (P/TAP-R) colony than in the allopatric colonies from Nuevo Leon and Guatemala. These two haplotypes might resemble the foothills population defined by microsatellites (f1/f2) [13]. Although similar recognition molecules were detected in the parasite-mosquito interactions, there may be parasite adaptations and selection driven by local mosquitoes. It is possible that these modifications are engendered by genetic flow and/or evolutionary convergence [42], perhaps a co-adaptation between the cspVk and P25/28 variants and diverse mosquito midgut binding molecules in regions where transmission is carried out by one predominantly abundant vector. As occurs in southern Mexico, the transmission of selective parasite strains by different mosquito species suggests a selective pressure generated by the vector-parasite interaction, as well as geographical isolation caused by the ecological conditions restricting mosquito breeding and distribution [38].

Although susceptibility patterns were similar between colonies of the same mosquito species, the highest infection rates and number of oocysts were produced by Vk247/Pvs25-B parasites in An. pseudopunctipennis, which might be related to the high parasitaemia, gametocytaemia and exflagellation rates detected in the blood infected with this versus other parasite haplotypes. Similarly, high parasitaemia was observed in patients from Brazil infected with $P$. vivax Vk247 [43]. The present results correspond partially to those reported in India with An. stephensi collected in the wild and fed with $P$. vivax infected blood, where the association between parasitaemia and the average number of oocysts was only subtle [44]. Given the low correlation, the degree of infectiveness of gametocytes (difficult to measure) together with certain mosquito factors may influence the establishment of infection and (to some extent) the number of oocysts. The only parameter herein associated with a lack of oocyst development was a low exflagellation rate.

The present findings suggest that distribution and dispersion of $P$. vivax haplotypes might depend on the competence of vector species, which is important for defining control measures. Malaria transmission in Mexico has been reduced to only a few foci and the country is in a pre-elimination stage. In the residual transmission foci, the resilience shown by the parasite in the face of malaria control efforts may owe itself to the high susceptibility of each anopheline species to specific parasite variants, likely similar to the parasite-vector combinations encountered in southern Mexico. The surveillance of possible parasite-reintroductions should be considered.

\section{Conclusions}

Anopheles albimanus and An. pseudopunctipennis, the main vectors of $P$. vivax in Mexico and Mesoamerica, transmit distinct $P$. vivax haplotypes and with different efficiency. In the various geographical sites herein tested, similarly higher susceptibility was found for An. albimanus to $P$. vivax haplotype cspVk/Pvs25-130 Vk210/ Pvs25-A than for An. pseudopunctipennis. This vector was highly susceptible to haplotypes Vk210/Pvs25-B and Vk247/Pvs25-B. The higher frequency of Vk247/Pvs25-B infections compared to other haplotypes of the parasite is in agreement with its higher parasitaemia and gametocytaemia. The present results also emphasize the importance of genotyping $P$. vivax to understand transmission dynamics and vector participation.

\section{Additional file}

Additional file 1: Table S1. Anopheles albimanus and An.

pseudopunctipennis: origin, collection and colonization, and generations used for P. vivax-feeding experiments. (DOC $46 \mathrm{~kb}$ )

\section{Abbreviations}

INSP: National Institute of Public Health; CRISP: Regional Centre for Research in Public Health; cspVk: circumsporozoite protein central repeat type; WS: white striped phenotype; TAP: Tapachula; AWS-R: Reference colony of An. albimanus; P/TAP-R: Reference colony of An. pseudopunctipennis

\section{Acknowledgments}

The authors thank all participating malaria patients who volunteered for this study. We also thank the insectary and the laboratory personnel for their technical assistance, especially to Marco A. Sandoval.

\section{Funding}

This work was funded by CONACYT-Mexico projects CB29005-M, CB31041-M and SALUD-2004-119.

\section{Availability of data and materials}

The datasets supporting the conclusions of this article are included within the article and its additional file. Raw data are available from the corresponding author upon request.

\section{Authors' contributions}

LGC and CVT conceived and designed the study. LGC was responsible for patient's management, all aspects of mosquito blood-feeding experiments, parasite counting and molecular analysis, participated in statistical analysis, preparation of figures and tables and drafted the manuscript. CVT was responsible for field collection of mosquitoes, establishment of mosquito colonies and supply, and drafted the manuscript. MHR participated in data analysis and drafted the manuscript. JANC participated in the experimental mosquito infections, mosquito dissections and parasite counting. JEHA participated in the statistical analysis and drafted the manuscript. IRMG participated in field collections, taxonomic identification and the rearing of mosquito species and colonies. FSV participated in molecular analysis and drafted the manuscript. All the authors read and approved the final version of the manuscript. 


\section{Ethics approval and consent to participate}

The present protocol was approved by the research and ethics committees of the National Institute of Public Health, Mexico (INSP). All participating adults gave informed consent. In the case of minors, informed consent was obtained from the parents or guardians prior to taking a blood sample.

\section{Consent for publication}

Not applicable.

\section{Competing interests}

The authors declare that there are no competing interests.

\section{Publisher's Note}

Springer Nature remains neutral with regard to jurisdictional claims in published maps and institutional affiliations.

\section{Author details}

${ }^{1}$ Centro Regional de Investigación en Salud Pública, Instituto Nacional de Salud Pública, Tapachula 30700, Chiapas, México. ${ }^{2}$ Centro de Investigación en Enfermedades Infecciosas, Instituto Nacional de Salud Pública, Cuernavaca 62100, Morelos, México. ${ }^{3}$ Centro de Información para Decisiones en Salud Pública, Instituto Nacional de Salud Pública, Ciudad de México 14080, México.

Received: 7 September 2018 Accepted: 31 January 2019 Published online: 20 February 2019

\section{References}

1. Zimmerman $\mathrm{RH}$. Ecology of malaria vectors in the Americas and future direction. Mem Inst Oswaldo Cruz. 1992;87(Suppl. 3):371-83.

2. Faran ME. Mosquito studies (Diptera, Culicidade) XXXIV. A revision of the albimanus section of the subgenus Nyssorhynchus of Anopheles. Contrib Am Entomol Inst. 1980;15:1-215.

3. Narang SK, Seawright JA, Suarez MF. Genetic structure of natural populations of Anopheles albimanus in Colombia. J Am Mosq Control Assoc. 1991;7:437-45

4. Beach RF, Mills D, Collins FH. Structure of ribosomal DNA in Anopheles albimanus (Diptera: Culicidae). Ann Entomol Soc Am. 1989;82:641-8.

5. Manguin S, Roberts DR, Peyton EL, Fernandez-Salas I, Barreto M, Fernandez Loayza R, et al. Biochemical systematics and population genetic structure of Anopheles pseudopunctipennis, vector of malaria in Central and South America. Am J Trop Med Hyg. 1995;53:362-77.

6. Organización Panamericana de la Salud. Informe de la situación de la malaria en las Américas, 2014. 2017. https://www.paho.org/hq/ dmdocuments/2016/2016-cha-informe-situacion-malaria-reg.pdf. Accessed 6 Nov 2018.

7. Secretaria de Salud. Boletín Epidemiológico Sistema Nacional de Vigilancia Epidemiológica Sistema Único de Información. https://www.gob.mx/salud/ documentos/boletinepidemiologico-sistema-nacional-de-vigilanciaepidemiologica-sistema-unico-de-informacion. Accessed 6 Nov 2018.

8. Arnot DE, Barnwell JW, Tam JP, Nussenzweig V, Nussenzweig RS, Enea V. Circumsporozoite protein of Plasmodium vivax: gene cloning and characterization of the immunodominant epitope. Science. 1985;230:815-8.

9. Rosenberg R, Wirtz RA, Lanar DE, Sattabongkot J, Hall T, Waters AP, et al. Circumsporozoite protein heterogeneity in the human malaria parasite Plasmodium vivax. Science. 1989;245:973-6.

10. Gonzalez-Ceron L, Rodriguez MH, Nettel JC, Villarreal C, Kain KC, Hernandez JE. Differential susceptibilities of Anopheles albimanus and Anopheles pseudopunctipennis to infections with coindigenous Plasmodium vivax variants VK210 and VK247 in southern Mexico. Infect Immun. 1999;67:410-2.

11. Carlton JM, Adams JH, Silva JC, Bidwell SL, Lorenzi H, Caler E, et al. Comparative genomics of the neglected human malaria parasite Plasmodium vivax. Nature. 2008;455:757-63.

12. Gonzalez-Ceron L, Alvarado-Delgado A, Martinez-Barnetche J, Rodriguez MH, Ovilla-Munoz M, Perez F, et al. Sequence variation of ookinete surface proteins Pvs 25 and Pvs 28 of Plasmodium vivax isolates from southern Mexico and their association to local anophelines infectivity. Infect Genet Evol. 2010;10:645-54

13. Joy DA, Gonzalez-Ceron L, Carlton JM, Gueye A, Fay M, McCutchan TF, et al. Local adaptation and vector-mediated population structure in Plasmodium vivax malaria. Mol Biol Evol. 2008;25:1245-52.
14. Collins WE, Skinner JC, Warren M, Richardson B. Studies on human malaria in Aotus monkey. VII. Comparative infectivity of two strains of Plasmodium vivax to Anopheles freeborni, A. maculatus, and for strains of A. albimanus. J Parasitol. 1976;62:190-4.

15. World Health Organization. Division of Malaria and Other Parasitic Diseases. Manual on practical entomology in malaria. 1995. http://www.who.int/iris/ handle/10665/42481. Accessed 6 Nov 2018.

16. Wilkerson RC, Strickman D, Litwak TR. Illustrated key to the female anopheline mosquitoes of Central America and Mexico. J Am Mosq Control Assoc. 1990;6:7-34.

17. Villarreal C, Arredondo-Jimenez JI, Rodriguez MH, Ulloa A. Colonization of Anopheles pseudopunctipennis from Mexico. J Am Mosq Control Assoc. 1998; 14:369-72.

18. Norma Oficial Mexicana NOM-062-ZOO-1999. Especificaciones técnicas para la producción, cuidado y uso de los animales de laboratorio. https://www. gob.mx/cms/uploads/attachment/file/203498/NOM-062-ZOO-1999_220801. pdf. Accessed 6 Nov 2018

19. Gonzalez-Ceron L, Rodriguez MH, Santillan FV, Hernandez JE, Wirtz RA. Susceptibility of three laboratory strains of Anopheles albimanus (Diptera: Culicidae) to coindigenous Plasmodium vivax circumsporozoite protein phenotypes in southern Mexico. J Med Entomol. 2000;37:331-4.

20. Norma Oficial Mexicana NOM-032-SSA2-2002. Para la vigilancia epidemiológica, prevención y control de enfermedades transmitidas por vector. 2002. http://www.salud.gob.mx/unidades/cdi/nom/032ssa202.html. Accessed 6 Nov 2018.

21. Carter R, Nijhout MM. Control of gamete formation (exflagellation) in malaria parasites. Science. 1977;195:407-9.

22. Gonzalez-Ceron L, Rodriguez MH, Betanzos AF, Abadia A. Efficacy of a rapid test to diagnose Plasmodium vivax in symptomatic patients of Chiapas. Mexico. Salud Publica Mex. 2005:47:282-7 (In Spanish).

23. Ogwan'g RA, Mwangi JK, Githure J, Were JB, Roberts CR, Martin SK. Factors affecting exflagellation of in vitro-cultivated Plasmodium falciparum gametocytes. Am J Trop Med Hyg. 1993;49:25-9.

24. Kruskal WH, Wallis WA. Use of ranks in one-criterion variance analysis. J Am Statist Assoc. 1952;47:583-621.

25. McCullangh P, Nelder JA. Generalized Linear Models. 2nd edition. London and Harpenden: Chapman and Hall; 1983.

26. Shao J, Tu D. The Jackknife and Bootstrap. New York: Springer; 1995.

27. Tukey JW. Bias and confidence in not-quite large sample. Ann Math Statist. 1958;29:614.

28. Spearman $C$. The proof and measurement of association between two things. Am J Psychol. 1904;15:72-101.

29. Ahumada ML, Orjuela LI, Pareja PX, Conde M, Cabarcas DM, Cubillos EF, et al. Spatial distributions of Anopheles species in relation to malaria incidence at 70 localities in the highly endemic northwest and South Pacific coast regions of Colombia. Malar J. 2016;15:407.

30. Gutiérrez LA, González JJ, Gómez GF, Castro MI, Rosero DA, Luckhart S, et al. Species composition and natural infectivity of anthropophilic Anopheles (Diptera: Culicidae) in the states of Córdoba and Antioquia, northwestern Colombia. Mem Inst Oswaldo Cruz. 2009;104:1117-24.

31. Lardeux F, Loayza P, Bouchité B, Chavez T. Host choice and human blood index of Anopheles pseudopunctipennis in a village of the Andean valleys of Bolivia. Malar J. 2007:6:8

32. Naranjo-Diaz N, Rosero DA, Rua-Uribe G, Luckhart S, Correa MM. Abundance, behavior and entomological inoculation rates of anthropophilic anophelines from a primary Colombian malaria endemic area. Parasit Vectors. 2013:6:61.

33. Tellaeche AM. La evolución y la situación actual del paludismo en México. In: Kumate J, Martinez-Palomo A, editors. A cien años del descubrimiento de Ross. El paludismo en Mexico. Mexico city: Colegio Nacional; 1988. p. 209-226.

34. Warren M, Collins WE, Jeffery GM, Richardson BB. Anopheles pseudopunctipennis: laboratory maintenance and malaria susceptibility of a strain from El Salvador. Am J Trop Med Hyg. 1980;29:503-6.

35. Young MD, Porter JA, Johnson CM. Plasmodium vivax transmitted from man to monkey to man. Science. 1966;153:1006-7.

36. Warren M, Mason J, Hobbs J. Natural infections of Anopheles albimanus with Plasmodium in a small malaria focus. Am J Trop Med Hyg. 1975;24:545-6.

37. Jeffery GM, Burgess RW, Eyles DE. Susceptibility of Anopheles quadrimaculatus and A. albimanus to domestic and foreign strains of Plasmodium vivax. Am J Trop Med Hyg. 1954;3:821-4. 
38. Li J, Collins WE, Wirtz RA, Rathore D, Lal A, McCutchan TF. Geographic subdivision of the range of the malaria parasite Plasmodium vivax. Emerg Infect Dis. 2001;7:35-42.

39. Achee NL, Korves CT, Bangs MJ, Rejmankova E, Lege M, Curtin D, et al. Plasmodium vivax polymorphs and Plasmodium falciparum circumsporozoite proteins in Anopheles (Diptera: Culicidae) from Belize. Central America. J Vector Ecol. 2000;25:203-11.

40. Gonzalez-Ceron L, Rodriguez MH, Santillan F, Chavez B, Nettel JA, Hernandez-Avila JE, et al. Plasmodium vivax: ookinete destruction and oocyst development arrest are responsible for Anopheles albimanus resistance to circumsporozoite phenotype VK247 parasites. Exp Parasitol. 2001;98:152-61.

41. Gonzalez-Ceron L, Rodriguez MH, Chavez-Munguia B, Santillan F, Nettel JA, Hernandez-Avila JE. Plasmodium vivax: impaired escape of Vk210 phenotype ookinetes from the midgut blood bolus of Anopheles pseudopunctipennis. Exp Parasitol. 2007;115:59-67.

42. Shi Y, Yokoyama S. Molecular analysis of the evolutionary significance of ultraviolet vision in vertebrates. Proc Natl Acad Sci USA. 2003;100:8308-13.

43. Ribeiro BdP, Cassiano GC, de Souza RM, Cysne DN, Grisotto MAG, de Azevedo dos Santos APS, et al. Polymorphisms in Plasmodium vivax circumsporozoite protein (CSP) influence parasite burden and cytokine balance in a pre-Amazon endemic area from Brazil. PLoS Negl Trop Dis. 2016;10:e0004479.

44. Balabaskaran Nina P, Mohanty AK, Ballav S, Vernekar S, Bhinge S, D'souza M, et al. Dynamics of Plasmodium vivax sporogony in wild Anopheles stephensi in a malaria-endemic region of Western India. Malar J. 2017;16:284.

Ready to submit your research? Choose BMC and benefit from:

- fast, convenient online submission

- thorough peer review by experienced researchers in your field

- rapid publication on acceptance

- support for research data, including large and complex data types

- gold Open Access which fosters wider collaboration and increased citations

- maximum visibility for your research: over $100 \mathrm{M}$ website views per year

At BMC, research is always in progress.

Learn more biomedcentral.com/submissions 